Pacific Journal of Mathematics

ADDITIONAL NOTE ON SOME TAUBERIAN THEOREMS OF 


\title{
ADDITIONAL NOTE ON SOME TAUBERIAN THEOREMS OF O. SZÁSZ
}

\author{
C. T. RAJAGOPAL
}

1. An additional theorem. In the note [3] to which this is an addition, Theorem II is exhibited as a generalization of Theorem I and an appeal is made to Szász [6] to indicate the transition from Theorem II to the final result stated as Corollary III'. However, in view of the formal simplicity of Corollary III' and the wide generality (reflected in its apparent complexity) of Theorem II, it seems worth while to adopt the opposite point of view and record a method, based on the following result, of deducing Theorem II and all related theorems (which cover Szász's) from Corollary III' [3, p. 384].

Theorem IV. If a (real) series $\sum_{n=1}^{\infty} a_{n}$ is $(\Phi, \lambda)$-summable to $s$, where $\lambda$ denotes the strictly positive increasing divergent sequence $\left\{\lambda_{n}\right\}$ subject to the additional condition $\lambda_{n+1} / \lambda_{n} \rightarrow 1$, and if the series satisfies the Tauberian condition:

$$
\liminf _{n \rightarrow \infty} \frac{1}{\lambda_{n}} \sum_{\nu=n+1}^{m} \lambda_{\nu} a_{\nu} \geq 0, \quad m>n, \frac{\lambda_{m}}{\lambda_{n}} \rightarrow 1,
$$

then $\sum_{n=1}^{\infty} a_{n}$ is convergent to s. (Amnon Jakimovski [1, Theorem 1] gives the case $\phi(u)=e^{-u}, \lambda_{n}=n$.)

Proof. We have, by Abel's partial-summation lemma,

$$
\sum_{\nu=n+1}^{m} a_{\nu}=\sum_{\nu=n+1}^{m} \frac{\lambda_{\nu} a_{\nu}}{\lambda_{\nu}} \geq \frac{\lambda_{n}}{\lambda_{n+1}} \cdot \frac{1}{\lambda_{n}} \min _{n+1 \leq k \leq m} \sum_{\nu=n+1}^{k} \lambda_{\nu} a_{\nu} .
$$

Hence, by (1),

$$
\underset{n \rightarrow \infty}{\lim \inf } \sum_{\nu=n+1}^{m} a_{\nu} \geq 0, \quad m>n, \quad \begin{aligned}
& \lambda_{m} \rightarrow 1 . \\
& \lambda_{n}
\end{aligned}
$$

It is well-known [2, p. 33] that the above Schmidt condition is equivalent to the second alternative of hypothesis (12) of Corollary III' $[3, p .384]$. Therefore this corollary establishes that $\sum_{n=1}^{\infty} a_{n}=s$.

\section{Deductions from Theorem IV.}

Corollary IV.1. In Theorem IV, (1) is implied by, and so can be replaced by, ONE of the following conditions:

Received August 4, 1954. 


$$
\left.\begin{array}{l}
\lim _{n \rightarrow \infty} \frac{1}{\lambda_{n}} \sum_{\nu=n+1}^{m} \lambda_{\nu}\left(\left|a_{\nu}\right|-a_{\nu}\right)=0, \\
\lim _{n \rightarrow \infty} \frac{1}{\lambda_{n}} \sum_{\nu=n+1}^{m} \lambda_{\nu}\left|a_{\nu}\right|=0,
\end{array}\right\} \quad m>n, \quad \lambda_{m}^{\lambda_{m} \rightarrow 1}
$$

(Szász [6, Theorem 3] gives the case $\phi(u)=e^{-u}, \lambda_{n}=n$.)

Corollary IV.2. In Corollary IV.1, (2) can be replaced by the condition:

$$
\begin{array}{lr}
U_{n} \equiv \sum_{\nu=1}^{n} \lambda_{\nu}\left(\left|a_{\nu}\right|-a_{\nu}\right)=O\left(\lambda_{n}\right), & n \rightarrow \infty, \\
\lim _{n \rightarrow \infty}\left(\begin{array}{c}
U_{m}-U_{n} \\
\lambda_{m}
\end{array}\right)=0 . & m>n, \begin{array}{l}
\lambda_{n} \\
\lambda_{m} \rightarrow 1 .
\end{array}
\end{array}
$$

(Szász [6, Theorem 2] gives the case $\phi(u)=e^{-u}, \lambda_{n}=n$.)

The above corollary is the same as Theorem II of my note [3]. We can deduce it from the preceding corollary merely by noting that (4) implies (2) ${ }^{1}$ as a result of letting $n \rightarrow \infty, \lambda_{m} / \lambda_{n} \rightarrow 1$ in the identity:

$$
\frac{U_{m}-U_{n}}{\lambda_{n}}=\left(\begin{array}{c}
U_{m} \\
\lambda_{m}
\end{array}-\frac{U_{n}}{\lambda_{n}}\right) \frac{\lambda_{m}}{\lambda_{n}}+\frac{U_{n}}{\lambda_{n}}\left(\begin{array}{c}
\lambda_{m} \\
\lambda_{n}
\end{array}-1\right), \quad m>n
$$

Corollary IV.3. In Corollary IV.1, (3) can be replaced by the hypothesis :

$$
\begin{array}{rr}
V_{n} \equiv \sum_{\nu=1}^{n} \lambda_{\nu}\left|a_{\nu}\right|=O\left(\lambda_{n}\right), & n \rightarrow \infty, \\
\lim _{n \rightarrow \infty}\left(\frac{V_{m}}{\lambda_{m}}-\frac{V_{n}}{\lambda_{n}}\right)=0, & m>n, \frac{\lambda_{m}}{\lambda_{n}} \rightarrow 1,
\end{array}
$$

which implies (3) exactly as (4) implies (2).

Plainly the last hypothesis (5) can assume the special form:

$$
\lim _{n \rightarrow \infty} \frac{V_{n}}{\lambda_{n}}=l
$$

(Szász and Rényi [6, Theorems 1 and B] give the particular case $\phi(u)=$ $e^{-u}, \lambda_{n}=n$.)

3. A second additional theorem. Theorem IV is a deduction from Corollary III' [3] and so ultimately from Theorem A [3, p. 378]. The following is another deduction from Theorem A deserving of mention.

${ }^{1}$ In fact (4) is equivalent to (2) as (2) implies (4) by an argument exactly like Szász's in the case $\lambda_{n}=n[\mathbf{6}$, Lemma 2]. 
Theorem B. Let $\phi(u)$ fulfill the conditions $\mathrm{C}(\mathrm{i})-(\mathrm{v})$ of the Introduction [3, p. 377]." Suppose that $A(u)$ is a (real) function of bounded variation in every finite interval of $(0, \infty), A(0)=0$. If

$$
\frac{1}{u} \int_{0}^{u} x d\{A(x)\}
$$

is slowly decreasing, that is,

$$
\liminf _{u \rightarrow \infty}\left(\frac{1}{v} \int_{0}^{v} x d\{A(x)\}-\frac{1}{u} \int_{0}^{u} x d\{A(x)\}\right) \geq 0, \quad v>u, \frac{v}{u} \rightarrow 1,
$$

and if $A(u)$ is $\Phi$-summable to $s$, that is, if

$$
\Phi(t)=\int_{0}^{\infty} \phi(u t) d\{A(u)\}
$$

exists for $t>0$ and tends to $s$ as $t \rightarrow+0$, then $A(u) \rightarrow s$ as $u \rightarrow \infty$.

Proof. We write as before [3, pp. 377-378]:

$$
A_{1}(u)=\int_{0}^{u} A(x) d x, \quad \phi(u)=\int_{u}^{\infty} \psi(x) d x .
$$

Then (7) gives successively [4, pp. 346-347], as $t \rightarrow+0$,

$$
\begin{gathered}
\Phi(t)=t \int_{0}^{\infty} \psi(u t) A(u) d u \rightarrow s, \quad \Phi_{1}(t)=t \int_{0}^{\infty} \psi(u t) \underset{u}{A_{1}(u)} d u \rightarrow s . \\
\Phi(t)-\Phi_{1}(t)=t \int_{0}^{\infty} \psi(u t)\left\{A(u)-u^{-1} A_{1}(u)\right\} d u \rightarrow 0 .
\end{gathered}
$$

Thus $A(u)-u^{-1} A_{1}(u)$ is $\Phi$-summable to 0 and satisfies the Tauberian condition in (6). Hence, by a known result [4, Corollary 2.2] following from Theorem A [3], $A(u)-u^{-1} A_{1}(u)$ tends to 0 as $u \rightarrow \infty$. Consequently, by Theorem A [3], $u^{-1} A_{1}(u)$, and hence also $A(u)$, tends to $s$ as $u \rightarrow \infty$.

4. Remarks. (i) Amnon Jakimovski [1, Theorem 1] has dealt with the case of Theorem B in which $\phi(u)=e^{-u}$ and

$$
A(u)=\left\{\begin{array}{c}
a_{1}+a_{2}+\cdots+a_{n} \text { for } n \leq u<n+1, n \geq 1, \\
0 \text { for } 0 \leq u<1,
\end{array}\right.
$$

showing, by a modification of the method used above to prove Theorem $B$, that we may in this case replace (6) by

$$
\liminf _{n \rightarrow \infty}\left(\frac{U_{m}^{*}}{m}-\begin{array}{c}
U_{n}^{*} \\
n
\end{array}\right) \geq 0, \quad m>n, \begin{gathered}
m \\
n
\end{gathered} \rightarrow 1,
$$

${ }^{2}$ These conditions can be slightly relaxed (for example, [5, Theorem A]). 
where $U_{n}^{*} \equiv \sum_{\nu=1}^{n} \nu a_{\nu}$, leaving the statement of Theorem B otherwise unaltered. He also observes that $\left(6^{*}\right)$ includes (or generalizes) the second half of (4) with $\lambda_{n}=n$, implying that, in Szász's result cited under Corollary IV.2, the first half of (4) is superfluous. This observation is, however, incorrect as shown by the following example.

Example 1. Let $a_{n}$ be defined so that

$$
\left.\begin{array}{ll}
n a_{n}=\nu & \text { for } \quad 4^{\nu} \leq n<2 \cdot 4^{\nu} \\
n a_{n}=-n^{-2} & \text { for } 2 \cdot 4^{\nu} \leq n<4^{\nu+1}
\end{array}, \quad\right\} \nu=0,1,2, \cdots .
$$

Then it is easily verified that (4) with $\lambda_{n}=n$ holds because

$$
\sum_{\nu=1}^{n} \nu\left(\left|a_{\nu}\right|-a_{\nu}\right)=o(n),
$$

but that $\left(6^{*}\right)$ does not hold since

$$
\text { if } n=2 \cdot 4^{\nu}, \nu \rightarrow \infty \text {, then } \frac{U_{n}^{*}}{n}=\frac{\sum_{k=0}^{\nu} k \cdot 4^{k}+O(1)}{2 \cdot 4^{\nu}} \sim \frac{2 \nu}{3},
$$

if $m=$ the integral part of $2 \cdot 4^{\nu}, \nu$, then $\underset{m}{U_{m}^{*}}=\underset{n}{U_{n}^{*}+o(1) \frac{n}{m}}$ where $(n / m-1) \sim-\nu^{-1 / 2}$, so that

$$
\liminf _{n \rightarrow \infty}\left(\begin{array}{c}
U_{m}^{*} \\
m
\end{array}-\begin{array}{c}
U_{n}^{*} \\
n
\end{array}\right)=-\infty, \quad \quad m>n, \frac{m}{n} \rightarrow 1 .
$$

While the above example shows that (4) with $\lambda_{n}=n$ does not in general imply $\left(6^{*}\right)$, the one which follows makes it clear that neither does $\left(6^{*}\right)$ necessarily imply (4) with $\lambda_{n}=n$.

ExAmple 2. Let $a_{n}$ be defined so that

$$
\left.\begin{array}{rl}
(-1)^{n} n a_{n} & =\nu \text { for } 4^{\nu} \leq n<2 \cdot 4^{\nu}, \\
a_{n} & =0 \text { for } 2 \cdot 4^{\nu} \leq n<4^{\nu+1},
\end{array}\right\} \nu=0,1,2, \cdots .
$$

Then $\left(6^{*}\right)$ holds since $U_{n}^{*} / n \rightarrow 0$ as $n \rightarrow \infty$. However, (4) with $\lambda_{n}=n$ does not hold since now

$$
U_{n}=\sum_{\nu=1}^{n} \nu\left(\left|a_{\nu}\right|-a_{\nu}\right)
$$

and we have:

$$
\text { if } n=2 \cdot 4^{\nu}, \nu \rightarrow \infty \text {, then } \begin{gathered}
U_{n} \\
2 n
\end{gathered}=\begin{gathered}
\sum_{k=1}^{\nu} k \cdot 4^{k} / 2 \\
2.4^{\nu}
\end{gathered}{ }_{3}^{\nu},
$$


if $m=$ the integral part of $2 \cdot 4^{\nu} \begin{gathered}\nu \\ \nu-V \nu\end{gathered}$, then $\begin{gathered}U_{m}=\begin{array}{l}U_{n} n \\ 2 m\end{array} 2 n m \\ 2 n m\end{gathered}$ where $(n / m-1) \sim-\nu^{-1 / 2}$, with the result that

$$
\liminf _{n \rightarrow \infty}\left(\frac{U_{m}}{m}-\frac{U_{n}}{n}\right)=-\infty, \quad m>n, \underset{n}{m} \rightarrow 1 .
$$

(ii) In the definition of $\Phi$-summability of $A(u)$, set forth in (7) and assumed in both Theorem A [3] and Theorem B, the integral $\Phi(t)$ is to be interpreted as a Lebesgue-Stieltjes integral (absolutely) convergent for $t>0$ unless further considerations, as in the case $\phi(u)=e^{-u}$, permit us to view it as a (non-absolutely) convergent Riemann-Stieltjes integral (cf. [5, p. 103, Note]).

(iii) In Theorem III [3, p. 383] the condition $\lambda_{n+1} / \lambda_{n} \rightarrow \infty$ of hypothesis (11) is a misprint for $\lambda_{n+1} / \lambda_{n} \rightarrow 1$.

In conclusion I wish to thank Dr. T. Vijayaraghavan ${ }^{3}$ and the referee for helpful suggestions.

\section{REFERENCES}

1. Amnon Jakimovski, On a Tauberian theorem by O. Szàsz, Proc. Amer. Math. Soc., 5 (1954), 67-70.

2. J. Karamata, Sur les théorèmes inverses des procédés de sommabilité, Actualités Sci. Ind., 450 (1937).

3. C. T. Rajagopal, A note on some Tauberian theorem of O. Szàsz, Pacific J. Math., 2 (1952), 377-384.

4. - A note on generalized Tauberian theorems, Proc. Amer. Math. Soc., 2 (1951), 335-349.

5. - Theorems on the produce of two summability methods with applications, J. Indian Math. Soc. (New Series), 18 (1954), 89-105.

6. O. Szász, On a Tauberian theorem for Abel summability, Pacific J. Math., 1 (1951), $117-125$.

RaMaNUJAN INSTITUTE of Mathematics

MADRAS, INDIA

3 Who passed into the beyond on April 20, 1955. 

.././. ./FrontMatter/paper .pdf 


\section{Pacific Journal of Mathematics}

Nesmith Cornett Ankeny and Theodore Joseph Rivlin, On a theorem of S.

Bernstei........................................ 849

Louis Auslander, The use of forms in variational calculation .......... 853

Paul Civin, Abstract Riemann sum . .......................... 861

Paul Civin, Some ergodic theorems involving two operator ............ 869

Eckford Cohen, The number of solutions of certain cubic congruence .... . 877

Richard M. Cohn, Specializations over difference field .............. 887

Jean Dieudonné, Pseudo-discriminant and Dickson invarian . . ......... 907

Ky Fan, A comparison theorem for eigenvalues of normal matrice ........ 911

Richard P. Gosselin, On the convergence behaviour of trigonometric interpolating polynomial ........................... 915

Peter K. Henrici, On generating functions of the Jacobi polynomial . . . . . . . 923

Meyer Jerison, An algebra associated with a compact grou ............ 933

Wilhelm Magnus, Infinite determinants associated with Hill's equatio . . . . . 941

G. Power and D. L. Scott-Hutton, The slow steady motion of liquid past a semi-elliptical bos................................. 953

Lyle E. Pursell, An algebraic characterization of fixed ideals in certain function ring .................................... 963

C. T. Rajagopal, Additional note on some Tauberian theorems of O. Szás . . 971 Louis Baker Rall, Error bounds for iterative solutions of Fredholm integral

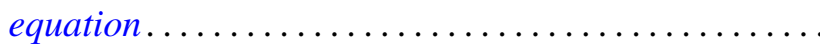

Shigeo Sasaki and Kentaro Yano, Pseudo-analytic vectors on

pseudo-Kählerian manifold ......................

Eugene Schenkman, On the tower theorem for finite group

P. Stein and John E. L. Peck, On the numerical solution of Poisson's equation over a rectangl ........................

Morgan Ward, The mappings of the positive integers into themselves which preserve divisio .

Seth Warner, Weak locally multiplicatively-convex algebra 1025

Louis Weisner, Group-theoretic origin of certain generating function .... 